\title{
$\lambda$-Semidirect products of inverse monoids are weakly Schreier extensions
}

\author{
Peter F. Faul ${ }^{1}$
}

Received: 16 May 2020 / Accepted: 17 June 2020 / Published online: 31 August 2020

(c) The Author(s) 2021

\begin{abstract}
A split extension of monoids with kernel $k: N \rightarrow G$, cokernel $e: G \rightarrow H$ and splitting $s: H \rightarrow G$ is weakly Schreier if each element $g \in G$ can be written $g=k(n) \operatorname{se}(g)$ for some $n \in N$. The characterization of weakly Schreier extensions allows them to be viewed as something akin to a weak semidirect product. The motivating examples of such extensions are the Artin glueings of topological spaces and, of course, the Schreier extensions of monoids which they generalise. In this paper we show that the $\lambda$-semidirect products of inverse monoids are also examples of weakly Schreier extensions. The characterization of weakly Schreier extensions sheds some light on the structure of $\lambda$-semidirect products. The set of weakly Schreier extensions between two monoids comes equipped with a natural poset structure, which induces an order on the set of $\lambda$-semidirect products between two inverse monoids. We show that Artin glueings are in fact $\lambda$-semidirect products and inspired by this identify a class of Artin-like $\lambda$-semidirect products. We show that joins exist for this special class of $\lambda$-semidirect product in the aforementioned order.
\end{abstract}

Keywords Semigroup · Artin gluing · Protomodular · Monoid extension · Lambda-semidirect products

\section{Introduction}

The ideas underlying the semidirect product of groups can be adapted to a number of structures. One such example is the context of semigroups wherein an action of semigroups $\alpha: H \times N \rightarrow N$ gives rise to a semidirect product $N \rtimes_{\alpha} H$ defined just as in the group case. These semidirect products have found much use in semigroup theory, for instance they provide some insight into the structure of inverse semigroups

Communicated by Victoria Gould.

$凶 \quad$ Peter F. Faul

peter@faul.io

1 Department of Pure Mathematics and Statistical Sciences, University of Cambridge, Cambridge, UK 
[9]. However, when applied naively to two inverse semigroups, this semidirect product does not in general yield an inverse semigroup. To remedy this Billhardt introduced a related notion called a $\lambda$-semidirect product [1]. Given inverse semigroups $N$ and $H$ the idea is to use an action of $H$ on $N$ to equip a certain subset of $N \times H$ (determined by the action) with a multiplication turning it into an inverse semigroup. These $\lambda$-semidirect products have since granted insight into the structure of inverse semigroups.

When we restrict this construction to inverse monoids we find that just as with groups, these $\lambda$-semidirect products naturally form a split extension. Here a split

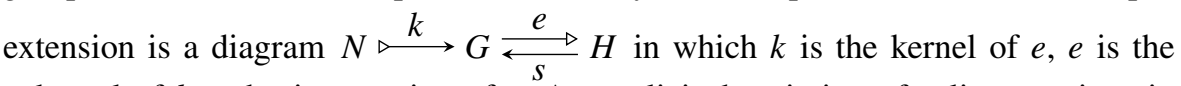
cokernel of $k$ and $s$ is a section of $e$. An explicit description of split extensions in the context of monoids is given just after Definition 2.1. Our restriction in this paper to inverse monoids may not be strictly necessary as there does exist a notion of a split extensions between general semigroups. These split extensions play a role in the structure theory of regular semigroups, analogous to the role $\lambda$-semidirect products play in the structure theory of inverse semigroups [8].

Semigroups are not the only context in which a generalization of the semidirect product can be considered. For monoids $N$ and $H$, an action $\alpha: H \times N \rightarrow N$ of $H$ on $N$ gives rise to a semidirect product $N \rtimes_{\alpha} H$ defined just as expected. In this context, as with groups, there is a relationship between semidirect products and split extensions [7]. Unlike in the case of groups where there is a one-to-one correspondence between semidirect products and split extensions, these semidirect products of monoids correspond to only the Schreier split extensions of monoids — those split extensions $N \triangleright \stackrel{k}{\longrightarrow} G \stackrel{e}{\stackrel{s}{\rightleftarrows}} H$ in which for each $g \in G$ there exists a unique $n \in N$ such that $g=k(n) \operatorname{se}(g)$.

The Schreier condition can be weakened and a new class of split extensions considered. Instead of requiring that for each $g \in G$ there is a unique $n \in N$, we can merely require that there exists (potentially many) $n \in N$ for which $g=k(n) s e(g)$. We call such an extension weakly Schreier and they were first considered in [2]. Their characterization in [3] establishes that they resemble something like a weak semidirect product. The primary (non-Schreier) examples of these extensions are the Artin glueings of topological spaces, where the lattices of open sets are viewed as monoids.

\section{Outline}

In this paper we will show that the $\lambda$-semidirect products of inverse monoids are also examples of weakly Schreier extensions. In fact this subsumes are previous example as we show that Artin glueings are in fact examples of $\lambda$-semidirect products. The characterization of weakly Schreier extensions sheds some light on $\lambda$-semidirect products. The set of weakly Schreier extensions between two monoids comes with a natural poset structure, which induces an order on the $\lambda$-semidirect products between two inverse monoids. The Artin glueing leads us to define a class of Artin-like $\lambda$ semidirect products. We show that this class is closed under binary joins. 


\section{Background}

In this section we give an introduction to the theory of weakly Schreier extensions of monoids, summarising the core results found in [3]. We then outline the basics of inverse semigroups and Billhardt's $\lambda$-semidirect products [1] before finally discussing frames and Artin glueings. In this paper we assume familiarity with the concept of a category and a functor, though a more comprehensive introduction to category theory can be found in [6].

\subsection{Weakly Schreier extensions}

The category Mon of monoids has zero morphisms between objects: the maps sending all elements to the identity. Consequently the kernel (dually the cokernel) of a morphism $f: A \rightarrow B$ can be defined in this category as the equaliser (dually the coequaliser) of $f$ and the zero morphism from $A$ into $B$. This allows us to define split extensions.

Definition 2.1 For monoids $H$ and $N$, a split extension of $N$ by $H$ is a diagram $N \stackrel{k}{\longleftrightarrow} G \stackrel{e}{\stackrel{S}{\rightleftarrows}} H$ in which $k$ is the kernel of $e, e$ is the cokernel of $k$, and $s$ is a section of $e-$ that is, $e s=1_{H}$.

Here the kernel $k$ is the equaliser of $e$ and the constant 1 map which in the context of monoids is the inclusion of all the elements sent by $e$ to 1 . The cokernel $e$ is the coequaliser of $k$ and the constant 1 map which is the quotient map given by the congruence generated from the pairs $(k(n), 1)$.

Split extensions are often considered in the category of groups where it is well known that they correspond to semidirect products. In Mon this relationship is not so simple. The appropriate notion of an action in Mon gives rise to a monoidal notion of a semidirect product. However, as discussed above, these are only in bijection with what are known as Schreier split extensions.

While this gives good motivation for these split extensions, one may ask if they are the only split extensions in Mon worth considering. As described in [4], there is a related class of split extensions that arises naturally from the world of topology. That specific example will be discussed in Sect. 2.3, but for now we consider the class of split extensions in question.

Definition 2.2 A split extension $N \stackrel{k}{\longrightarrow} G \stackrel{e}{\stackrel{S}{\rightleftarrows}} H$ is weakly Schreier if for each $g \in G$ there exists $n \in N$ such that $g=k(n) \operatorname{se}(g)$.

Here, compared to Schreier extensions, we have dropped the uniqueness requirement on the $n \in N$ and this influences our ability to think of them as semidirect products.

Intuitively, when we have a split extension $N \stackrel{k}{\longleftrightarrow} G \stackrel{e}{\stackrel{s}{\rightleftarrows}} H$ of groups, there is a bijection between the set $N \times H$ and $G$ which sends the pair $(n, h)$ to $k(n) s(h)$. When 
we let the set $N \times H$ inherit a multiplication through this bijection we arrive at the associated semidirect product. This is why Schreier extensions are desirable in Mon, as they ensure this bijection exists and allows the same inheritance of multiplication.

In both cases these semidirect products can be characterized by actions which encode this inherited multiplication. If $\alpha: H \times N \rightarrow N$ is an action of $H$ on $N$, then the multiplication is given by $(n, h) \cdot\left(n^{\prime}, h^{\prime}\right)=\left(n \alpha\left(h, n^{\prime}\right), h h^{\prime}\right)$.

For weakly Schreier extensions there is no bijection between the elements of $N \times H$ and $G$, instead we have a surjective map sending $(n, h)$ to $k(n) s(h)$. We do, however, get a bijection between $G$ and the quotient of $N \times H$ given by this surjection. This quotient will then inherit a multiplication from $G$ and it is this object that we call the associated weak semidirect product.

In terms of characterizing these weak semidirect products, it is no longer enough to just specify an action, as there is now this quotient to consider. In addition, the presence of this quotient means that we no longer need an action to specify the multiplication - something weaker will do.

Definition 2.3 For monoids $N$ and $H$, an equivalence relation on $N \times H$ is said to be admissible if the following conditions hold.

(1) $(n, 1) \sim\left(n^{\prime}, 1\right)$ implies $n=n^{\prime}$,

(2) $(n, h) \sim\left(n^{\prime}, h^{\prime}\right)$ implies $h=h^{\prime}$,

(3) $\left(n_{1}, h\right) \sim\left(n_{2}, h\right)$ implies $\left(x n_{1}, h\right) \sim\left(x n_{2}, h\right)$ for all $x \in N$,

(4) $\left(n_{1}, h\right) \sim\left(n_{2}, h\right)$ implies $\left(n_{1}, h y\right) \sim\left(n_{2}, h y\right)$ for all $y \in H$.

Quotients arising from admissible equivalence relations are precisely the quotients that occur in weak semidirect products.

Definition 2.4 A function $\alpha: H \times N \rightarrow N$ is an action compatible with an admissible equivalence relation $E$ on $N \times H$ if it satisfies the following conditions:

(1) $\left(n_{1}, h\right) \sim\left(n_{2}, h\right)$ implies $\left(n_{1} \alpha(h, n), h\right) \sim\left(n_{2} \alpha(h, n), h\right)$ for all $n \in N$,

(2) $\left(n, h^{\prime}\right) \sim\left(n^{\prime}, h^{\prime}\right)$ implies $\left(\alpha(h, n), h h^{\prime}\right) \sim\left(\alpha\left(h, n^{\prime}\right), h h^{\prime}\right)$ for all $h \in H$,

(3) $\left(\alpha\left(h, n n^{\prime}\right), h\right) \sim\left(\alpha(h, n) \cdot \alpha\left(h, n^{\prime}\right), h\right)$,

(4) $\left(\alpha\left(h h^{\prime}, n\right), h h^{\prime}\right) \sim\left(\alpha\left(h, \alpha\left(h^{\prime}, n\right)\right), h h^{\prime}\right)$,

(5) $(\alpha(h, 1), h) \sim(1, h)$,

(6) $(\alpha(1, n), 1) \sim(n, 1)$.

Conditions (3)-(6) are reminiscent of the usual action definition, except that in this case they are only required to hold up to equivalence.

These are essentially all the needed ingredients to characterize weak semidirect products. The only wrinkle is that two actions compatible with an admissible equivalence relation can sometimes induce the same multiplication on the quotient. Thus instead of considering the set $\operatorname{Act}_{E}(H, N)$ of actions compatible with $E$, we consider a quotient $\operatorname{Act}_{E}(H, N) / \sim$ where two actions $\alpha$ and $\beta$ are equivalent if $(\alpha(h, n), h) \sim(\beta(h, n), h)$ for all $n \in N$ and $h \in H$. See [3] for more details.

Proposition 2.5 For monoids $N$ and $H$, let $E$ be an admissible equivalence relation and $\alpha$ a compatible action. Then $(E,[\alpha])$ corresponds to a weakly Schreier extension

$$
N \triangleright \stackrel{k}{\longrightarrow}(N \times H / E, \cdot,[1,1]) \stackrel{e}{\stackrel{S}{\rightleftarrows}} H
$$


in which $k(n)=[n, 1], e([n, h])=h$ and $s(h)=[1, h]$. Multiplication is defined as

$$
[n, h] \cdot\left[n^{\prime}, h^{\prime}\right]=\left[n \alpha\left(h, n^{\prime}\right), h h^{\prime}\right]
$$

Similarly we can consider a reverse of this process.

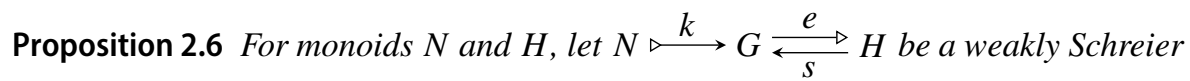
extension and let $q: G \rightarrow N$ be a function such that $g=k q(g) \operatorname{se}(g)$. Then we can associate to this a pair $(E,[\alpha])$ where $E$ is an admissible equivalence relation defined by

$$
(n, h) \sim\left(n^{\prime}, h^{\prime}\right) \Longleftrightarrow k(n) s(h)=k\left(n^{\prime}\right) s\left(h^{\prime}\right) .
$$

and $\alpha$ is a compatible action defined by

$$
\alpha(h, n)=q(s(h) k(n)) .
$$

Notice that there must exist such a map $q$ by virtue of the split extension being weakly Schreier (using the axiom of choice). The choice of $q$ does not end up mattering.

These two processes are inverses of one another up to isomorphism. Let us discuss what the morphisms in question are.

Definition 2.7 A morphism of weakly Schreier extensions is a monoid homomorphism $f: G_{1} \rightarrow G_{2}$ such that the three squares in the following diagram commute.

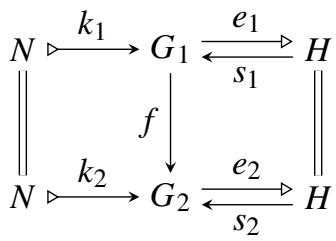

If $f: G_{1} \rightarrow G_{2}$ is such a morphism, then for all $g \in G_{1}$, there exists an $n \in N$ such that $f(g)=f\left(k_{1}(n) \cdot s_{1} e_{1}(g)\right)=f k_{1}(n) \cdot f s_{1}\left(e_{1}(g)\right)=k_{2}(n) \cdot s_{2}\left(e_{1}(g)\right)$. This means that any morphism between two weakly Schreier extensions must be unique.

Proposition 2.8 The category WSExt $(H, N)$ of weakly Schreier extensions between $N$ and $H$ and morphisms of weakly Schreier extensions is a preorder.

Inspired by the above we can define an order relation on our pairs $(E,[\alpha])$. Let

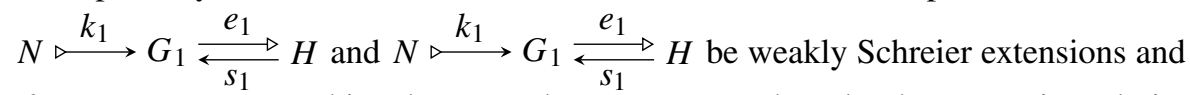
$f: G_{1} \rightarrow G_{2}$ a morphism between them. Let $E_{1}$ and $E_{2}$ be the respective admissible equivalence relations and $q_{1}$ and $q_{2}$ associated Schreier retractions. Then our above calculation implies that $f\left([n, h]_{E_{1}}\right)=[n, h]_{E_{2}}$. This will only be well-defined when $(n, h) \sim_{E_{1}}\left(n^{\prime}, h\right)$ implies $(n, h) \sim_{E_{2}}\left(n^{\prime}, h\right)$. The fact that $f$ must preserve multiplication is equivalent to the statement that $\left(q_{1}\left(s_{1}(h)\left(k_{1}(n)\right)\right), h\right) \sim_{E_{2}}$ $\left(q_{2}\left(s_{2}(h) k_{2}(n)\right), h\right)$. Thus we can define the order as follows. 
Definition 2.9 Let $\operatorname{WAct}(H, N)$ have as objects pairs $(E,[\alpha])$ where $E$ is an admissible equivalence relation on $N \times H$ and $\alpha$ is a compatible action. Then we say $\left(E_{1},\left[\alpha_{1}\right]\right) \leq\left(E_{2},\left[\alpha_{2}\right]\right)$ if and only if $(n, h) \sim_{E_{1}}\left(n^{\prime}, h\right)$ implies $(n, h) \sim_{E_{2}}\left(n^{\prime}, h\right)$ and for all $n \in N$ and $h \in H\left(\alpha_{1}(h, n), h\right) \sim_{E_{2}}\left(\alpha_{2}(h, n), h\right)$.

Using the transformations provided in Propositions 2.5 and 2.6 we get the following equivalence.

Theorem 2.10 The categories $\operatorname{WSExt}(H, N)$ and $\operatorname{WAct}(H, N)$ are equivalent.

\subsection{Inverse semigroups and $\lambda$-semidirect products}

Definition 2.11 A semigroup $S$ is called an inverse semigroup when for each $x \in S$ there exists a unique element $x^{-1} \in S$ such that $x x^{-1} x=x$ and $x^{-1} x x^{-1}=x^{-1}$.

Given an inverse semigroup $S$ and an element $x \in S$ it can be easily seen that $x x^{-1}$ and $x^{-1} x$ are idempotents. Furthermore in an inverse semigroup it is always the case that idempotents commute with one another. For a comprehensive introduction to the subject, see [5].

As discussed above, the standard semigroup semidirect product construction, when applied to two inverse semigroups, will not in general return an inverse semigroup. Thus, we study Billhardt's $\lambda$-semidirect product [1]. The idea is to consider an algebraic structure on a subset of the product of two inverse semigroups.

Definition 2.12 Let $N$ and $H$ be inverse semigroups and let $\alpha: H \times N \rightarrow N$ be a function which we write as $\alpha(h, n)=h \cdot n$. Then $\alpha$ is an action of inverse semigroups if the following conditions are satisfied for all $h, h^{\prime} \in H$ and $n, n^{\prime} \in N$.

(1) $h \cdot\left(n n^{\prime}\right)=(h \cdot n)\left(h \cdot n^{\prime}\right)$,

(2) $h h^{\prime} \cdot n=h \cdot\left(h^{\prime} \cdot n\right)$.

An action could, of course, equivalently be defined as a homomorphism from $H$ into the endomorphisms of $N$.

Definition 2.13 Let $N$ and $H$ be inverse semigroups and let $H$ act on $N$. Then the $\lambda$-semidirect product associated to this action has as underlying set

$$
\left\{(n, h) \in N \times H: h h^{-1} \cdot n=n\right\}
$$

and multiplication defined by

$$
\left(n_{1}, h_{1}\right)\left(n_{2}, h_{2}\right)=\left(\left(\left(h_{1} h_{2}\right)\left(h_{1} h_{2}\right)^{-1} \cdot n_{1}\right)\left(h_{1} \cdot n_{2}\right), h_{1} h_{2}\right)
$$

This multiplication resembles the multiplication of the standard semidirect product in a number of ways. The only disagreement is that instead of $\left(h \cdot n_{2}\right)$ being multiplied on the left by $n_{1}$, it is being multiplied on the left by $\left(h_{1} h_{2}\right)\left(h_{1} h_{2}\right)^{-1} \cdot n_{1}$. 


\subsection{Frames and Artin glueings}

A motivating example of weakly Schreier extensions is the Artin glueing of frames. As we shall see in this section, Artin glueings have some interesting parallels to $\lambda$ semidirect products.

A frame is an algebraic structure that captures the lattice of open sets of a topological space. A frame has finite meet operations capturing finite intersections of open sets, and arbitrary joins corresponding to arbitrary unions of opens. Finally we require meets distribute over arbitrary joins. For a more comprehensive look at frames, see [10].

Definition 2.14 A frame $L$ is a poset with finite meets and arbitrary joins such that finite meets distribute over joins.

We treat frames as algebraic structures and so the morphisms are just the maps preserving this structure.

Definition 2.15 A morphism $f: L \rightarrow M$ of frames satisifies

(1) $f(0)=0$,

(2) $f(1)=1$,

(3) $f(a \wedge b)=f(a) \wedge f(b)$,

(4) $f(\bigvee S)=\bigvee f(S)$.

Given a continuous map between two topological spaces, we know that the preimage sends opens to opens and from set theoretic properties of the preimage, preserves the empty set, the whole space, finite intersections and arbitrary unions. That is, the preimage is a frame homomorphism between the corresponding lattices of open sets.

This idea gives rise to a contravariant functor from the category of topological spaces to the category of frames. Furthermore, the category of frames is seen to be a subcategory of the category of monoids, where a frame $(L, \wedge, \vee, 1,0)$ is thought of as the monoid $(L, \wedge, 1)$. (To see that this is injective on objects note that the finite meet operation determines the order structure and consequently the joins). Note that it is not a full subcategory, as general monoid homomorphisms need not preserve the joins. Thus, we obtain a functor from the category of topological spaces into the category of monoids.

Transporting topological spaces into the category of monoids gives a worthwhile perspective on the well-known Artin glueing construction (see [11]). An Artin glueing of two topological spaces $N$ and $H$ is a topological space in which $N$ embeds as a closed subspace and $H$ as its open complement. For any two spaces there are in general many distinct Artin glueings and each is determined by a finite-meet-preserving map from $\mathcal{O}(H)$ to $\mathcal{O}(N)$. We present this construction below in the context of frames.

Definition 2.16 Let $N$ and $H$ be frames and $f: H \rightarrow N$ be a (finite-)meet-preserving map. Then the Artin glueing $\operatorname{Gl}(f)$ is the frame of pairs $(n, h)$ for which $n \leq f(h)$ with componentwise meets and joins.

In [4] it was shown that Artin glueings precisely correspond to the weakly Schreier extensions in the full subcategory of Mon consisting of frames. 
We see immediately some similarities with $\lambda$-semidirect products. The Artin glueing associates to a map an algebraic structure on a subobject of the product. Furthermore, the condition $n \leq f(h)$ is equivalent to $n \wedge f(h)=n$. We will revisit this idea in Sect. 5 where we will show that Artin glueings are in fact $\lambda$-semidirect products.

\section{$3 \lambda$-Semidirect products of inverse monoids}

In order to relate the $\lambda$-semidirect product to weakly Schreier extensions of monoids, we must work inside the category of monoids. Thus, in this section we consider only inverse monoids - that is, inverse semigroups with a unit.

In order to consider $\lambda$-semidirect products in this context there is one standard modification that is made to the theory, relating to the definition of an action.

Definition 3.1 Let $N$ and $H$ be inverse monoids and let $\alpha: H \times N \rightarrow N$ be a function with application written $\alpha(h, n)=h \cdot n$. Then $\alpha$ is an action of inverse monoids if it is an action of inverse semigroups and satisifies that for all $n \in N$

$$
1 \cdot n=n
$$

Notably, it is not required that $h \cdot 1=1$. Thus, the action can equivalently be thought of as a monoid homomorphism into the monoid of semigroup endomorphisms of $N$.

The $\lambda$-semidirect products we consider in this context are only taken with respect to actions of inverse monoids, as these are precisely the actions for which the associated $\lambda$-semidirect product is a monoid. (The pair $(1,1)$ acts as identity.)

Proposition 3.2 Let $N$ and $H$ be inverse monoids and let $\alpha: H \times N \rightarrow N$ be an action of inverse monoids. If $N \rtimes_{\alpha} H$ is the associated $\lambda$-semidirect product, then the following functions are monoid homomorphisms.

(1) $k: N \rightarrow N \rtimes_{\alpha} H$, where $k(n)=(n, 1)$,

(2) $e: N \rtimes_{\alpha} H \rightarrow H$, where $e(n, h)=h$,

(3) $s: H \rightarrow N \rtimes_{\alpha} H$, where $s(h)=\left(h h^{-1} \cdot 1, h\right)$.

Proof (1) We begin by proving that the function is well defined. This entails showing that $1\left(1^{-1}\right) \cdot n=n$. Since the inverse of 1 is 1 we use the fact that $\alpha$ is an action of inverse monoids.

Next observe that

$$
\begin{aligned}
k\left(n_{1}\right) k\left(n_{2}\right) & =\left(n_{1}, 1\right)\left(n_{2}, 1\right) \\
& =\left(\left(1\left(1^{-1}\right) \cdot n_{1}\right)\left(1 \cdot n_{2}\right), 1\right) \\
& =\left(n_{1} n_{2}, 1\right) \\
& =k\left(n_{1} n_{2}\right) .
\end{aligned}
$$

It is clear the unit is preserved. 
(2) The function is automatically well defined and it is very easy to see that it preserves the multiplication and unit.

(3) Again we begin by proving it is well defined. We must show that $\left(h h^{-1}\right) \cdot\left(h h^{-1}\right.$. 1) $=h h^{-1} \cdot 1$. This follows from the fact that $\alpha$ is action of semigroups and that $h h^{-1}$ is an idempotent.

Finally observe the following calculation.

$$
\begin{aligned}
s\left(h_{1}\right) s\left(h_{2}\right) & =\left(h_{1} h_{1}^{-1} \cdot 1, h_{1}\right)\left(h_{2} h_{2}^{-1} \cdot 1, h_{2}\right) \\
& =\left(\left(\left(h_{1} h_{2}\right)\left(h_{1} h_{2}\right)^{-1} \cdot h_{1} h_{1}^{-1} \cdot 1\right)\left(h_{1} \cdot h_{2} h_{2}^{-1} \cdot 1\right), h_{1} h_{2}\right) \\
& =\left(\left(h_{1} h_{2} h_{2}^{-1} h_{1}^{-1} h_{1} h_{1}^{-1} \cdot 1\right)\left(h_{1} h_{2} h_{2}^{-1} \cdot 1\right), h_{1} h_{2}\right) \\
& =\left(h_{1} h_{2} h_{2}^{-1} \cdot\left(\left(h_{1}^{-1} h_{1} h_{1}^{-1} \cdot 1\right)(1)\right), h_{1} h_{2}\right) \\
& =\left(h_{1} h_{2} h_{2}^{-1} h_{1}^{-1} \cdot 1, h_{1} h_{2}\right) \\
& =s\left(h_{1} h_{2}\right) .
\end{aligned}
$$

Where the transition from the third line to the fourth involves factoring out $h_{1} h_{2} h_{2}^{-1}$ from both terms.

Finally, note that $s(1)=\left(1\left(1^{-1}\right) \cdot 1,1\right)=(1 \cdot 1,1)=(1,1)$, the identity.

It is apparent that $k$ is the kernel of $e$ and that $s$ splits $e$. Below we show that this diagram is indeed a weakly Schreier extension.

Proposition 3.3 Let $N$ and $H$ be inverse monoids, $\alpha: H \times N \rightarrow N$ an action of inverse monoids, $N \rtimes_{\alpha} H$ the associated $\lambda$-semidirect product and let $k$, $e$ and $s$ be as in Proposition 3.2. Then $N \bowtie \stackrel{k}{\longleftrightarrow} N \rtimes_{\alpha} H \underset{S}{\stackrel{e}{\rightleftarrows}} H$ is a weakly Schreier extension.

Proof As discussed, it is apparent that $k$ is the kernel and $s$ is the splitting of $e$. Thus, we must only show that $e$ is the cokernel of $k$ and that the weakly Schreier condition holds. We begin with the latter. Let $(n, h) \in N \rtimes_{\alpha} H$ and consider

$$
\begin{aligned}
k(n) s(h) & =(n, 1)\left(h h^{-1} \cdot 1, h\right) \\
& =\left(\left(h h^{-1} \cdot n\right)\left(1 \cdot h h^{-1} \cdot 1\right), h\right) \\
& =\left(h h^{-1} \cdot n, h\right) \\
& =(n, h) .
\end{aligned}
$$

Here the last line follows because $(n, h)$ was assumed to belong to $S \rtimes_{\alpha} T$.

To see that $e$ is the cokernel consider a map $t: N \rtimes_{\alpha} H \rightarrow X$ such that $t k$ is the zero morphism. We must show that there is a unique map $\ell: H \rightarrow X$ such that $t=\ell e$.

By the above $t(n, h)=t(k(n) s(h))=t s(h)$. We then need only observe that for $\ell=t s$ we have $\ell e(n, h)=t s(h)$, as required. Since $e$ has a splitting, it is epic and consequently the map $\ell=t s$ must be unique.

Since $\lambda$-semidirect products of inverse monoids $N$ and $H$ are weakly Schreier extensions, we can view them instead as a particular admissible quotient of $N \times H$ paired with a compatible action. 


\subsection{The admissible quotient and compatible action}

Let $\alpha$ be an action of inverse monoids of $H$ on $N$ and let $N \stackrel{k}{\longleftrightarrow} N \rtimes_{\alpha} H \stackrel{e}{\rightleftarrows} H$ be the weakly Schreier extension corresponding to the associated $\lambda$-semidirect product. Then two pairs $\left(n_{1}, h_{1}\right)$ and $\left(n_{1}, h_{2}\right)$ will be related in the admissible quotient if and only if $k\left(n_{1}\right) s\left(h_{1}\right)=k\left(n_{2}\right) s\left(h_{2}\right)$. This amounts to requiring that $h_{1}=h_{2}$ and that $h_{1} h_{1}^{-1} \cdot n_{1}=h_{1} h_{1}^{-1} \cdot n_{2}$.

Proposition 3.4 Let $N \stackrel{k}{\longleftrightarrow} N \rtimes_{\alpha} H \stackrel{e}{\stackrel{S}{\rightleftarrows}} H$ be the weakly Schreier extension corresponding to a $\lambda$-semidirect product. Then $(n, h) \sim\left(h h^{-1} \cdot n, h\right)$ in the associated admissible equivalence relation.

Proof Since the second components agree, we need only verify that $h h^{-1} \cdot n=h h^{-1}$. $h h^{-1} \cdot n$. This follows from $\alpha$ being an action of semigroups and from the idempotence of $h h^{-1}$.

This means that each equivalence class $[n, h]$ has a canonical representative $\left(h h^{-1}\right.$. $n, h)$. The set of these representatives is easily seen to be the underlying set of $S \rtimes_{\alpha} T$.

In order to determine a compatible action we first consider the associated Schreier retraction. It is easy to see that the first projection $\pi_{1}: N \rtimes_{\alpha} H \rightarrow N$ is such a map. (Recall that the Schreier retraction need not be monoid homomorphisms). Given this Schreier retraction the compatible action is thus $\beta: H \times N \rightarrow N$ where

$$
\begin{aligned}
\beta(h, n) & =\pi_{1}(s(h) k(n)) \\
& =\pi_{1}\left(\left(h h^{-1} \cdot 1, h\right)(n, 1)\right) \\
& =\pi_{1}\left(\left(h h^{-1} \cdot h h^{-1} \cdot 1\right)(h \cdot n), h\right) \\
& =\left(h h^{-1} \cdot 1\right)(h \cdot n) \\
& =\left(h h^{-1} \cdot 1\right)\left(h h^{-1} h \cdot n\right) \\
& =h h^{-1} \cdot(1(h \cdot n)) \\
& =h \cdot n .
\end{aligned}
$$

Thus, the compatible action $\beta$ is just the original action $\alpha$.

Recall that from the weakly Schreier perspective the multiplication is given by

$$
\left[n_{1}, h_{1}\right]\left[n_{2}, h_{2}\right]=\left[n_{1}\left(h_{1} \cdot n_{1}\right), h_{1} h_{2}\right]
$$

The element $\left(n_{1}\left(h_{1} \cdot n_{2}\right), h_{1} h_{2}\right)$ will not in general be the canonical element of its class. Thus, we pass to the canonical element and arrive at

$$
\begin{aligned}
& \left(h_{1} h_{2}\left(h_{1} h_{2}\right)^{-1} \cdot\left(n_{1}\left(h_{1} \cdot n_{2}\right), h_{1} h_{2}\right)\right. \\
& \quad=\left(\left(h_{1} h_{2}\left(h_{1} h_{2}\right)^{-1} \cdot n_{1}\right)\left(h_{1} h_{2}\left(h_{1} h_{2}\right)^{-1} \cdot h_{1} \cdot n_{2}\right), h_{1} h_{2}\right) \\
& \quad=\left(\left(h_{1} h_{2}\left(h_{1} h_{2}\right)^{-1} \cdot n_{1}\right)\left(h_{1} \cdot\left(h_{2} h_{2}^{-1} \cdot n_{2}\right)\right), h_{1} h_{2}\right) .
\end{aligned}
$$


Note that if $\left(n_{2}, h_{2}\right) \in N \rtimes_{\alpha} H$, then the expression reduces to $\left(\left(h_{1} h_{2}\left(h_{1} h_{2}\right)^{-1}\right.\right.$. $\left.\left.n_{1}\right)\left(h_{1} \cdot n_{2}\right), h_{1} h_{2}\right)$ which is precisely the multiplication of $N \rtimes_{\alpha} H$.

\section{The preorder of $\lambda$-semidirect products}

Since the set of weakly Schreier extensions between monoids $N$ and $H$ has a natural preorder structure, we can now ask what order this induces on the set of $\lambda$-semidirect products when we take $N$ and $H$ to be inverse monoids.

It will be convenient to think in terms of the actions of inverse monoids instead of the $\lambda$-semidirect products themselves. Thus, we consider the preorder induced on the set of actions by the function sending an action to its associated weakly Schreier extension.

This function is not injective as two distinct actions can be mapped to isomorphic weakly Schreier extensions.

Example 1 Let $N$ be an inverse monoid with at least two distinct idempotents $u$ and $u^{\prime}$ and let $H$ be an inverse semigroup satisfying that $h_{1} h_{2}=1$ implies $h_{1}=1=h_{2}$.

Consider the function $\alpha_{u}: H \times N \rightarrow N$ where $\alpha_{u}(h, n)=u$ whenever $h \neq 1$ and $\alpha_{u}(1, n)=n$. Because $h_{1} h_{2}=1$ implies $h_{1}=1=h_{2}$ we have that $\alpha_{u}$ is an action of inverse monoids.

Similarly, consider the action $\alpha_{u^{\prime}}: H \times N \rightarrow N$ where $\alpha_{u^{\prime}}(h, n)=u^{\prime}$ whenever $h \neq 1$ and $\alpha_{u^{\prime}}(1, n)=n$.

It is apparent that $\alpha_{u} \neq \alpha_{u^{\prime}}$. Furthermore, both actions result in an equivalence relation in which $\left(n_{1}, h\right) \sim\left(n_{2}, h\right)$ for all $n_{1}, n_{2} \in N$ and $h \in H-\{1\}$, and $\left(n_{1}, 1\right) \sim$ $\left(n_{2}, 1\right)$ if and only if $n_{1}=n_{2}$. The multiplications agree as required, as in both equivalence relations we have that $\left(\alpha_{u}(h, n), h\right) \sim\left(\alpha_{u^{\prime}}(h, n), h\right)$.

Proposition 4.1 Let $N$ and $H$ be inverse monoids and let $\alpha: H \times N \rightarrow N$ and $\beta: H \times N \rightarrow N$ be actions of inverse monoids. Then $\alpha \leq \beta$ if and only if for all $n \in N$ and $h \in H, \beta\left(h h^{-1}, \alpha(h, n)\right)=\beta(h, n)$.

Proof $(\Rightarrow)$ Suppose that $\alpha \leq \beta$.

Then $(\alpha(h, n), h) \sim_{\beta}(\beta(h, n), h)$. Unwinding this gives

$$
\begin{aligned}
\beta\left(h h^{-1}, \alpha(h, n)\right) & =\beta\left(h h^{-1}, \beta(h, n)\right) \\
& =\beta(h, n) .
\end{aligned}
$$

$(\Leftarrow)$ Suppose that for all $n \in N$ and $h \in H, \beta\left(h h^{-1}, \alpha(h, n)\right)=\beta(h, n)$.

First we show that $\left(n_{1}, h\right) \sim_{\alpha}\left(n_{2}, h\right)$ implies $\left(n_{1}, h\right) \sim_{\beta}\left(n_{2}, h\right)$. Suppose that $\left(n_{1}, h\right) \sim_{\alpha}\left(n_{2}, h\right)$. This means that $\alpha\left(h h^{-1}, n_{1}\right)=\alpha\left(h h^{-1}, n_{2}\right)$. Thus, making use of our assumption we find 


$$
\begin{aligned}
\beta\left(h h^{-1}, n_{1}\right) & =\beta\left(h h^{-1}\left(h h^{-1}\right)^{-1}, \alpha\left(h h^{-1}, n_{1}\right)\right) \\
& =\beta\left(h h^{-1}, \alpha\left(h h^{-1}, n_{1}\right)\right) \\
& =\beta\left(h h^{-1}, \alpha\left(h h^{-1}, n_{2}\right)\right) \\
& =\beta\left(h h^{-1}, n_{2}\right) .
\end{aligned}
$$

Now we must show that $(\alpha(h, n), h) \sim_{\beta}(\beta(h, n), h)$. For these to be related we need that $\beta\left(h h^{-1}, \alpha(h, n)\right)=\beta\left(h h^{-1}, \beta(h, n)\right)$. By assumption $\beta\left(h h^{-1}, \alpha(h, n)\right)=$ $\beta(h, n)$ and combined with the fact that $\beta(h, n)=\beta\left(h h^{-1}, \beta(h, n)\right)$, we obtain the desired equality.

\section{Artin-glueing-like actions}

Given an order structure on the set of $\lambda$-semidirect products, it is natural to consider if meets and joins exist. In the section we show that joins exist for a natural class of $\lambda$-semidirect products, reminiscent of Artin glueings of frames.

As alluded to in the introduction, Artin glueings of frames are nothing more than a certain class of $\lambda$-semidirect products between certain meet-semilattices.

Proposition 5.1 Let $N$ and $H$ be frames considered in the category of monoids and let $f: H \rightarrow N$ be a monoid homomorphism. Then the Artin glueing $\operatorname{Gl}(f)$ is a $\lambda$-semidirect product of $N$ by $H$.

Proof The action corresponding to $\mathrm{Gl}(f)$ is given by $\alpha(h, n)=f(h) \wedge n$. Let us begin by confirming that this is an action of inverse monoids.

It is clear that $\alpha(1, n)=n$ as $f$ preserves the identity. Next observe

$$
\begin{aligned}
\alpha\left(h, n \wedge n^{\prime}\right) & =f(h) \wedge n \wedge n^{\prime} \\
& =f(h) \wedge n \wedge f(h) \wedge n^{\prime} \\
& =\alpha(h, n) \wedge \alpha\left(h, n^{\prime}\right) .
\end{aligned}
$$

Finally consider

$$
\begin{aligned}
\alpha\left(h \wedge h^{\prime}, n\right) & =f\left(h \wedge h^{\prime}\right) \wedge n \\
& =f(h) \wedge f\left(h^{\prime}\right) \wedge n \\
& =\alpha\left(h, f\left(h^{\prime}\right) \wedge n\right) \\
& =\alpha\left(h, \alpha\left(h^{\prime}, n\right)\right) .
\end{aligned}
$$

Thus, it remains only to show that $N \rtimes_{\alpha} H=\mathrm{Gl}(f)$.

Since the inverse of an element in a meet semilattice is itself and because of idempotence, we have that the elements of $N \rtimes_{\alpha} H$ are those pairs $(n, h)$ in which $n=f(h) \wedge n$. These are precisely the pairs in which $n \leq f(h)$ and so $N \rtimes_{\alpha} H$ and $\operatorname{Gl}(f)$ agree on elements. 
Using the same properties of meet-semilattices we see that the multiplication in $N \rtimes_{\alpha} H$ is given by

$$
\begin{aligned}
(n, h)\left(n^{\prime}, h^{\prime}\right) & =\left(\left(f(h) \wedge f\left(h^{\prime}\right) \wedge n\right) \wedge\left(f(h) \wedge n^{\prime}\right), h \wedge h^{\prime}\right) \\
& =\left(f(h) \wedge n \wedge f\left(h^{\prime}\right) \wedge n^{\prime}, h \wedge h^{\prime}\right) \\
& =\left(n \wedge n^{\prime}, h \wedge h^{\prime}\right) .
\end{aligned}
$$

This coincides with the multiplication of $\mathrm{Gl}(f)$ and so we are done.

As discussed in [4], if $N$ and $H$ are frames and $f, g: H \rightarrow N$ are monoid homomorphisms, then $\mathrm{Gl}(f \wedge g)$ is the join of $\mathrm{Gl}(f)$ and $\mathrm{Gl}(g)$ in the order structure on Artin glueings. In fact, as we shall see, $\mathrm{Gl}(f \wedge g)$ is the join of $\mathrm{Gl}(f)$ and $\mathrm{Gl}(g)$ in $\operatorname{WSExt}(H, N)$.

Inspired by the above, we would like to consider actions $\alpha$ of inverse monoids such that $\alpha(h, n)=f(h) \cdot n$ where $f$ is some function from $H$ into $N$. The condition that $\alpha$ be an action precludes many functions $f$ from serving this purpose. It is sufficient for $f$ to factor through the central idempotents of $S$.

Proposition 5.2 Let $H$ and $N$ be inverse monoids and let $f: H \rightarrow E(N) \cap Z(N)$ be a monoid homomorphism into the central idempotents of $N$, where $E(N)$ denotes the idempotents of $N$ and $Z(N)$ the central elements. Then $\alpha(h, n)=f(h) \cdot n$ is an action of inverse monoids.

Proof For $\alpha\left(h, n_{1} n_{2}\right)$ we have

$$
\begin{aligned}
\alpha\left(h, n_{1} n_{2}\right) & =f(h) \cdot n_{1} n_{2} \\
& =f(h) f(h) \cdot n_{1} n_{2} \\
& =f(h) n_{1} \cdot f(h) n_{2} \\
& =\alpha\left(h, n_{1}\right) \alpha\left(h, n_{2}\right),
\end{aligned}
$$

which makes use of the fact that $f(h)$ is a central idempotent.

Next we must check that $\alpha\left(h_{1} h_{2}, n\right)=\alpha\left(h_{1}, \alpha\left(h_{2}, n\right)\right)$. Here we consider

$$
\begin{aligned}
\alpha\left(h_{1} h_{2}, n\right) & =f\left(h_{1} h_{2}\right) \cdot n \\
& =f\left(h_{1}\right) f\left(h_{2}\right) \cdot n \\
& =f\left(h_{1}\right) \cdot \alpha\left(h_{2}, n\right) \\
& =\alpha\left(h_{1}, \alpha\left(h_{2}, n\right)\right) .
\end{aligned}
$$

The final condition follows easily with $\alpha(1, n)=f(1) \cdot n=1 \cdot n=n$.

Definition 5.3 Let $H$ and $N$ be inverse monoids and $f: H \rightarrow E(N) \cap Z(N)$ a monoid homomorphism into the central idempotents of $N$. Then we call the action $\alpha_{f}(h, n)=f(h) \cdot n$ the Artin-like-action corresponding to $f$. 
The $\lambda$-semidirect products resulting from Artin-like-actions have many nice properties. For instance, when interpreted as a weakly Schreier extensions, the canonical element of each equivalence class can be easily seen to be the smallest element in each class.

Furthermore, just as in the frame setting, we can combine two actions of this form in a natural way.

Proposition 5.4 Let $N$ and $H$ be inverse semigroups and let $\alpha_{f}, \alpha_{g}$ be Artin-likeactions corresponding to the maps $f, g: H \rightarrow E(N) \cap Z(N)$ respectively. Then the action $\gamma: H \times N \rightarrow N$ given by $\gamma(h, n)=f(h) g(h) n$, is an Artin-like-action.

Proof We claim that $\gamma$ corresponds to $\alpha_{f \cdot g}$, where $f \cdot g(h)=f(h) g(h)$. It is clear that $f \cdot g$ preserves the identity. To see that it preserves multiplication we make use of the fact that both $f$ and $g$ map into the centre of $N$. Thus we have

$$
\begin{aligned}
f \cdot g\left(h_{1} h_{2}\right) & =f\left(h_{1} h_{2}\right) g\left(h_{1} h_{2}\right) \\
& =f\left(h_{1}\right) f\left(h_{2}\right) g\left(h_{1}\right) g\left(h_{2}\right) \\
& =f\left(h_{1}\right) g\left(h_{1}\right) f\left(h_{2}\right) g\left(h_{2}\right) \\
& =f \cdot g\left(h_{1}\right) f \cdot g\left(h_{2}\right)
\end{aligned}
$$

and can conclude that $f \cdot g$ is a monoid homomorphism as required.

We then invoke Proposition 5.2 and we are done.

Proposition 5.5 Let $N$ and $H$ be inverse monoids and let $f, g: H \rightarrow E(N) \cap Z(N)$ be monoid homomorphisms into the central idempotents of $N$. Then the join of $\alpha_{f}$ and $\alpha_{g}$ exists in $\operatorname{WSExt}(H, N)$ and is equal to $\alpha_{f \cdot g}$.

Proof First we show that $\alpha_{f \cdot g}$ is larger than $\alpha_{f}$ and $\alpha_{g}$ in $\operatorname{WSExt}(H, N)$.

If $\left(n_{1}, h\right) \sim_{\alpha_{f}}\left(n_{2}, h\right)$ then $f(h) n_{1}=f(h) n_{2}$. Thus, $g(h) f(h) n_{1}=g(h) f(h) n_{2}$ and since $g(h)$ is central, we have $f g(h) n_{1}=f g(h) n_{2}$. This means that $\left(n_{1}, h\right) \sim_{\alpha_{f \cdot g}}$ $\left(n_{2}, h\right)$ as required. This same argument gives that $\left(n_{1}, h\right) \sim_{\alpha_{g}}\left(n_{2}, h\right)$ implies that $\left(n_{1}, h\right) \sim_{\alpha_{f \cdot g}}\left(n_{2}, h\right)$.

The final condition to check is that $(g(h) n, h) \sim_{\alpha_{f \cdot g}}(f g(h) n, h) \sim_{\alpha_{f \cdot g}}(f(h) n, h)$. This follows because $f(h)$ and $g(h)$ are both central and idempotent.

To show that $\alpha_{f \cdot g}$ is the join suppose we have a weakly Schreier extension $(E, \beta)$ larger than $\alpha_{f}$ and $\alpha_{g}$, but smaller than $\alpha_{f \cdot g}$. Since $(E, \beta)$ is smaller than $\alpha_{f \cdot g}$, we have that if $\left(n_{1}, h\right) \sim_{E}\left(n_{2}, h\right)$ then $\left(n_{1}, h\right) \sim_{\alpha_{f \cdot g}}\left(n_{2}, h\right)$. We will show that $(E, \beta)$ being larger than $\alpha_{f}$ and $\alpha_{g}$ means that $\left(n_{1}, h\right) \sim_{\alpha_{f \cdot g}}\left(n_{2}, h\right)$ implies that $\left(n_{1}, h\right) \sim_{E}\left(n_{2}, h\right)$.

We know that $(g(h) n, h) \sim_{E}(n, h) \sim_{E}(f(h) n, h)$ for all $n \in N$ and $h \in H$. Now suppose that $\left(n_{1}, h\right) \sim_{\alpha_{f \cdot g}}\left(n_{2}, h\right)$. This means that $f(h) g(h) n_{1}=f(h) g(h) n_{2}$. Now 
simply consider

$$
\begin{aligned}
(n, h) & \sim_{E}\left(f(h) n_{1}, h\right) \\
& \sim_{E}(f(h), 1)\left(n_{1}, h\right) \\
& \sim_{E}(f(h), 1)\left(g(h) n_{1}, h\right) \\
& \sim_{E}\left(f(h) g(h) n_{1}, h\right) \\
& \sim_{E}\left(f(h) g(h) n_{2}, h\right) \\
& \sim_{E}\left(n_{2}, h\right) .
\end{aligned}
$$

Thus, the equivalence relations are equal and so $(E, \beta)=\alpha_{f \cdot g}$.

$$
\text { Notice that this gives that } \mathrm{Gl}(f \wedge g)=\mathrm{Gl}(f) \vee \mathrm{Gl}(g) \text { in } \operatorname{WSExt}(H, N) \text {. }
$$

Open Access This article is licensed under a Creative Commons Attribution 4.0 International License, which permits use, sharing, adaptation, distribution and reproduction in any medium or format, as long as you give appropriate credit to the original author(s) and the source, provide a link to the Creative Commons licence, and indicate if changes were made. The images or other third party material in this article are included in the article's Creative Commons licence, unless indicated otherwise in a credit line to the material. If material is not included in the article's Creative Commons licence and your intended use is not permitted by statutory regulation or exceeds the permitted use, you will need to obtain permission directly from the copyright holder. To view a copy of this licence, visit http://creativecommons.org/licenses/by/4.0/.

\section{References}

1. Billhardt, B.: On a wreath product embedding and idempotent pure congruences on inverse semigroups. Semigroup Forum 45(1), 45-54 (1992)

2. Bourn, D.: Partial Mal'tsevness and partial protomodularity. Preprint arXiv:1507.02886v1 (2015)

3. Faul, P.F.: A characterization of weakly Schreier extensions of monoids. arXiv preprint arXiv:1911.02630 (2019)

4. Faul, P.F., Manuell, G.R.: Artin glueings of frames as semidirect products. J. Pure Appl. Algebra 224(8), 106334 (2020)

5. Lawson, M.V.: Inverse Semigroups: the Theory of Partial Symmetries. World Scientific, Singapore (1998)

6. Mac Lane, S.: Categories for the Working Mathematician. Springer, Berlin (1971)

7. Martins-Ferreira, N., Montoli, A., Sobral, M.: Semidirect products and crossed modules in monoids with operations. J. Pure Appl. Algebra 217(2), 334-347 (2013)

8. McAlister, D., Blyth, T.: Split orthodox semigroups. J. Algebra 51(2), 491-525 (1978)

9. McAlister, D.B.: Groups, semilattices and inverse semigroups. II. Trans. Am. Math. Soc. 196, 351-370 (1974)

10. Picado, J., Pultr, A.: Frames and Locales: Topology Without Points. Springer, Berlin (2011)

11. Wraith, G.: Artin glueing. J. Pure Appl. Algebra 4(3), 345-348 (1974)

Publisher's Note Springer Nature remains neutral with regard to jurisdictional claims in published maps and institutional affiliations. 